\title{
Scanning the past
}

\author{
Heinrich Rohrer and the scanning tunnelling microscope.
}

Heinrich Rohrer, the co-inventor of the scanning tunnelling microscope (STM), died on 16 May 2013. He was 79. Together with Gerd Binnig, he developed an instrument capable of imaging and manipulating individual atoms and molecules, and played a crucial role in the emergence of the field of nanoscience and technology.

The STM creates images by scanning a sharp metallic tip across the surface of a conducting material. The tip is placed close enough to the surface for the electron wavefunctions of the atoms in the tip to overlap with the wavefunctions of the atoms in the surface (a separation of less than $1 \mathrm{~nm}$ ). When a voltage is applied between the tip and the surface, electrons can quantum mechanically tunnel between the two. This tunnelling current depends exponentially on the distance between the tip and the surface. Therefore, by measuring the variations in the current as the tip is scanned across a sample (or, more typically, the variations in the height of the tip at a constant tunnelling current), a picture of the atomic corrugation of the surface can be built up line-by-line.

Rohrer was born on 6 June 1933 in Buchs, a village in eastern Switzerland. He studied physics at the Swiss Federal Institute of Technology in Zurich, graduating in 1955, and completed a PhD on superconductivity at the same institution in 1960. Following a postdoctoral research fellowship at Rutgers University in New Jersey, he joined IBM's Zurich Research Laboratory in 1963 and hired Binnig from Frankfurt University in 1978.

Rohrer and Binnig first successfully tested the STM on 16 March 1981. The initial paper on their results, which reported controlled vacuum tunnelling and was co-authored with Christoph Gerber and Edi Weibel, was published in the journal Applied Physics Letters in January $1982^{1}$. (The work was originally submitted to another leading physics journal in June 1981, but rejected on the basis of some mixed referee reports ${ }^{2}$.) The paper concludes on an optimistic note, suggesting that the technique "should open the door to a new area of surface studies."

The IBM team quickly followed this work with a report on the atomic-scale imaging of $\mathrm{CaIrSn}_{4}$ and gold surfaces ${ }^{3}$. However, it was the imaging of the $7 \times 7$ surface reconstruction of $\mathrm{Si}(111)$ in 1983 that really caught the attention of other scientists ${ }^{4}$. The exact details of this reconstruction, which occurs when the surface is heated to high

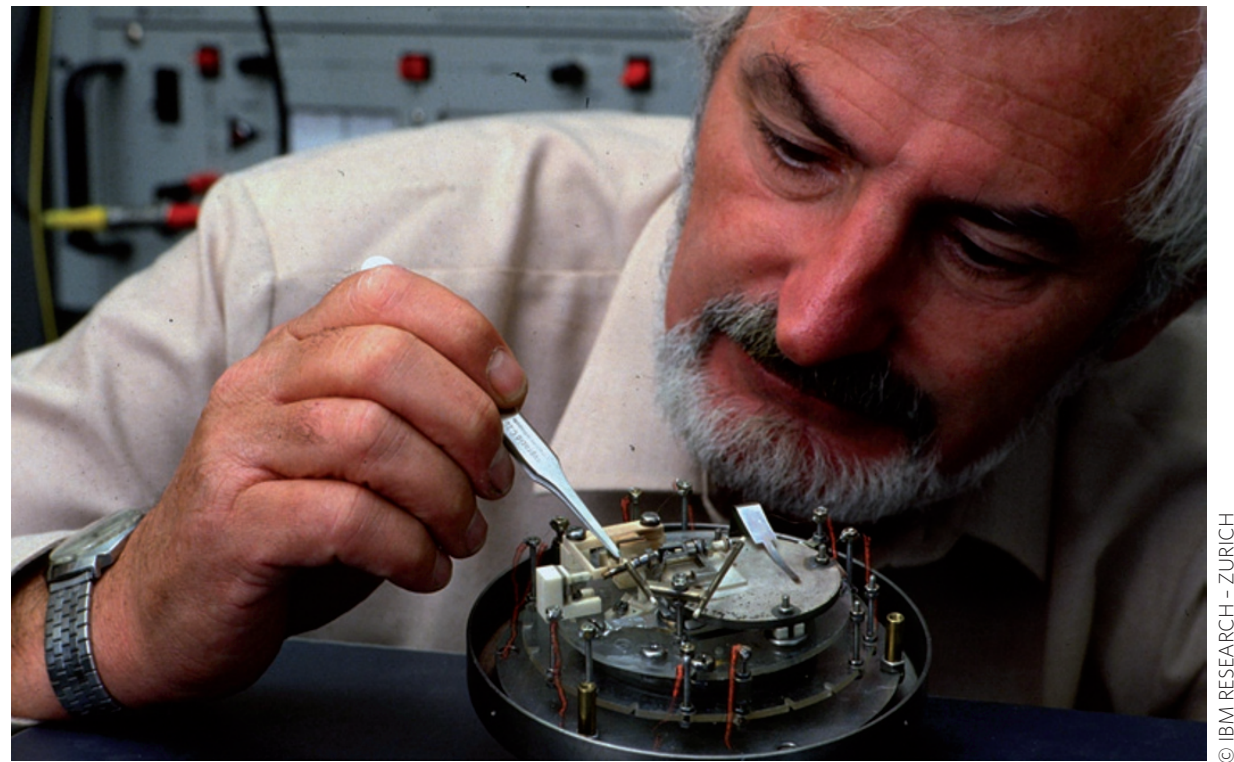

temperature, had been a topic of fascination for many in the surface science community and direct imaging of the surface with the STM helped to resolve the structural mystery.

Only 5 years after their initial experiments, Rohrer and Binnig were awarded the Nobel Prize in Physics for the design of the STM. (The prize was shared with Ernst Ruska, who had invented the electron microscope in 1933 and, in contrast, had to wait 53 years for the prize.) In these first few years, the focus was on using the STM to image surfaces. However, Rohrer and Binnig included in their Nobel Prize lecture ${ }^{5}$ a brief note on the possibility of using the instrument as a 'Feynman Machine': a reference to Richard Feynman's vision of controlling individual atoms and molecules.

Shortly after the duo received their Nobel Prize, Russell Becker, Jene Golovchenko and Brian Swartzentruber at Bell Labs reported using the tip of an STM to modify a germanium surface ${ }^{6}$. However, it was Don Eigler and Erhard Schweizer, working at IBM's Almaden Research Center in San Jose, who, in April 1990, provided the most profound demonstration of atomic manipulation with the STM ${ }^{7}$. They used the instrument to spell out 'IBM' with 35 xenon atoms on a nickel surface and created what are probably the most famous images in the history of nanotechnology. Three years later, Eigler, together with Michael Crommie and Christopher Lutz, created quantum corrals by moving iron atoms on a copper surface ${ }^{8}$.
The STM has since yielded numerous scientific and technological insights, and been used to analyse an ever-increasing variety of samples. Yet, there is, of course, another significant consequence of Rohrer and Binnig's discovery - it spawned an array of other scanning probe microscopes. The most notable of these is the atomic force microscope (AFM), which was invented by Binnig, Calvin Quate and Gerber in $1986^{\circ}$.

The AFM is now found in more laboratories than the STM, and is used to tackle a more diverse array of topics, particularly in biology. Work with scanning probe microscopes is also only a slice of what constitutes nanotechnology research today. However, the STM arguably remains the instrument most emblematic of the field, and Rohrer - together with the likes of Feynman, who died in 1988, and Richard Smalley, who died in 2005 - will forever be linked with the birth of nanotechnology.

References

1. Binnig, G., Rohrer, H., Gerber, Ch. \& Weibel, E. Appl. Phys. Lett. 40, 178-180 (1982).

2. Gerber, C. \& Lang, H. P. Nature Nanotech. 1, 3-5 (2006).

3. Binnig, G., Rohrer, H., Gerber, Ch. \& Weibel, E. Phys. Rev. Lett. 49, 57-61 (1982).

4. Binnig, G., Rohrer, H., Gerber, Ch. \& Weibel, E. Phys. Rev. Lett. 50, 120-123 (1983)

5. Binnig, G. \& Rohrer, H. Rev. Mod. Phys. 59, 615-625 (1987)

6. Becker, R., Golovchenko, J. \& Swarzentruber, B. Nature 325, 419-421 (1987).

. Eigler, D. M. \& Schweizer, E. K. Nature 344, 524-526 (1990).

8. Crommie, M. F., Lutz, C. P. \& Eigler, D. M. Science 262, 218-220 (1993).

9. Binnig, G., Quate, C. F. \& Gerber, Ch. Phys. Rev. Lett. 56, 930-933 (1986). 